\title{
Is BAC Transgenesis Obsolete? State of the Art in the Era of Designer Nucleases
}

\author{
J. Beil, ${ }^{1}$ L. Fairbairn, ${ }^{1}$ P. Pelczar, ${ }^{2}$ and T. Buch ${ }^{1}$ \\ ${ }^{1}$ Institute for Medical Microbiology, Immunology and Hygiene, Technische Universität München, Trogerstraße 30, \\ 81679 Munich, Germany \\ ${ }^{2}$ Institute of Animal Laboratory Sciences, VetSuisse Faculty, University of Zurich, Winterthurer Straße 190, \\ 8057 Zurich, Switzerland \\ Correspondence should be addressed to T. Buch, thorsten.buch@tum.de \\ Received 10 April 2012; Accepted 31 May 2012 \\ Academic Editor: Masamitsu Yamaguchi
}

Copyright (๑) 2012 J. Beil et al. This is an open access article distributed under the Creative Commons Attribution License, which permits unrestricted use, distribution, and reproduction in any medium, provided the original work is properly cited.

DNA constructs based on bacterial artificial chromosomes (BACs) are frequently used to generate transgenic animals as they reduce the influence of position effects and allow predictable expression patterns for genes whose regulatory sequences are not fully identified. Despite these advantages BAC transgenics suffer from drawbacks such as complicated vector construction, low efficiency of transgenesis, and some remaining expression variegation. The recent development of transcription activator-like effector nucleases (TALENs) and zinc finger nucleases (ZFNs) has resulted in new transgenic techniques which do not have the drawbacks associated with BAC transgenesis. Initial reports indicate that such designer nucleases (DNs) allow the targeted insertion of transgenes into endogenous loci by direct injection of the targeting vector and mRNA/DNA encoding the predesigned nucleases into oocytes. This results in the transgene being inserted at a specific locus in the mouse genome, thus circumventing the drawbacks associated with BAC transgenesis.

Sophisticated transgenic mouse models frequently require that the transgene expression is restricted to a particular tissue or cell type as defined by a specific promoter. BACs have been the most commonly used method for generation of transgenic animals when the transcriptional control elements of the gene of interest have not been clearly identified. Thus, in many applications BACs replaced "conventional" transgenesis using short constructs with well-defined promoter regions. BACs are based on the single-copy functional fertility plasmid (F factor) of Escherichia coli. In contrast to high copy number plasmids, replication of the $\mathrm{F}$ factor is tightly controlled and the plasmid exists as only one or two copies per cell. Unidirectional replication is determined by the regulatory genes oriS and repE of the $\mathrm{F}$ plasmid while the copy number is controlled by parA and parB genes. These regulatory elements, together with a resistance marker, constitute the BAC vector backbone that facilitates cloning and stable propagation of DNA fragments up to 300 kilobase pairs [1]. Originally developed for construction of genomic libraries used in the early genome sequencing projects, $\mathrm{BAC}$ constructs and $\mathrm{BAC}$ libraries have also proven to be very useful genetic tools in other aspects of basic research.

As mentioned before, the use of BACs enabled scientists to generate transgenes even when the promoter region of a gene is unknown. Furthermore, BAC-based transgenes more frequently yield the expected expression pattern among founder lines compared to conventional transgenes. This is most likely the result of reduced influence of position effects due to either the sheer size of the BAC transgenes which insulate the transgene cassette from the influence of the chromosomal environment or through the inclusion of elements such as enhancers, silencers, locus control regions, and matrix attachment regions. BAC transgenes can be rapidly constructed through recombination in E. coli and subsequently used to generate transgenic animals using standard techniques such as pronuclear injection in case of mice or rats (Figure 1(a)). This rapid and relatively straight 


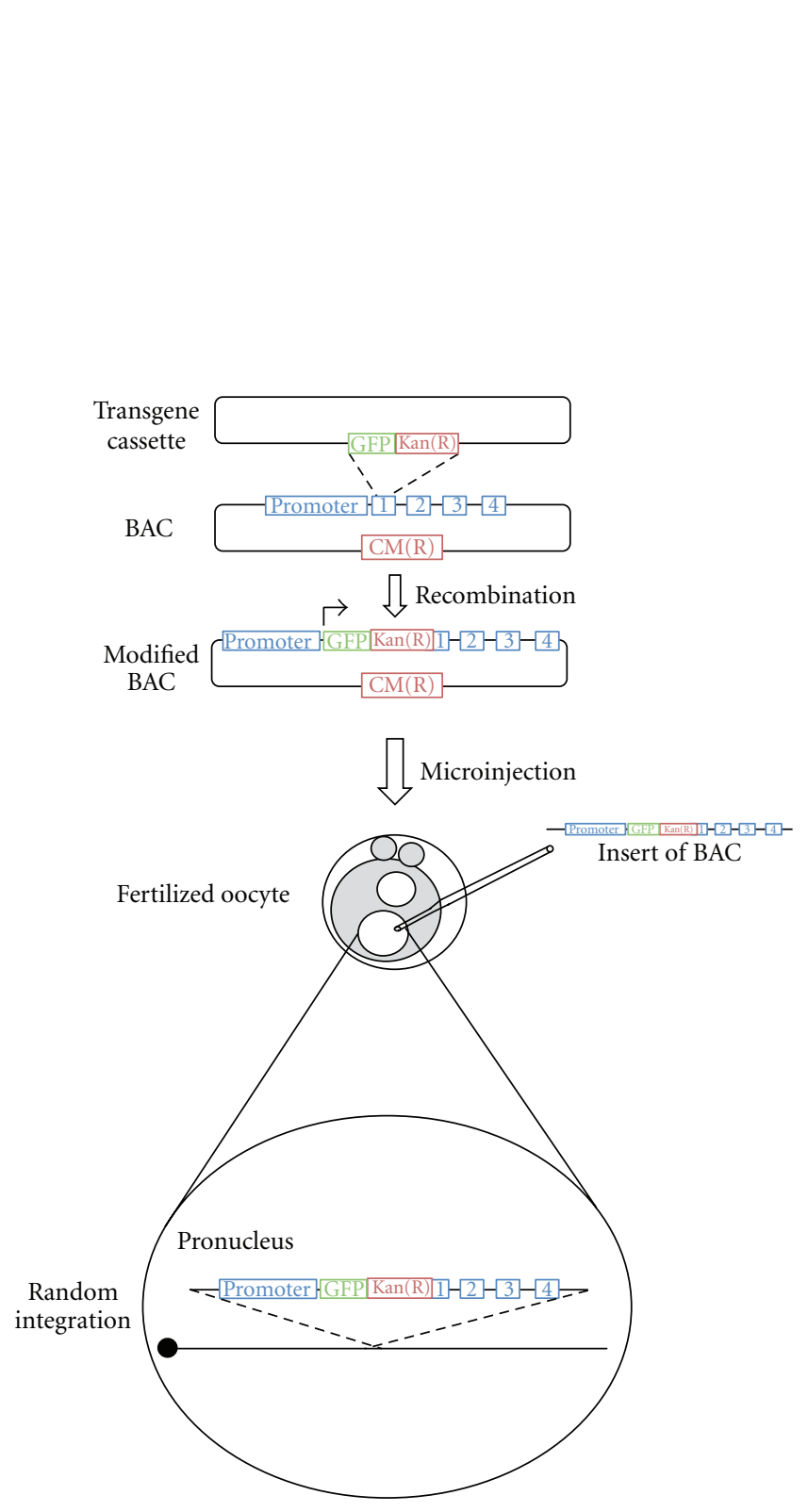

(a)
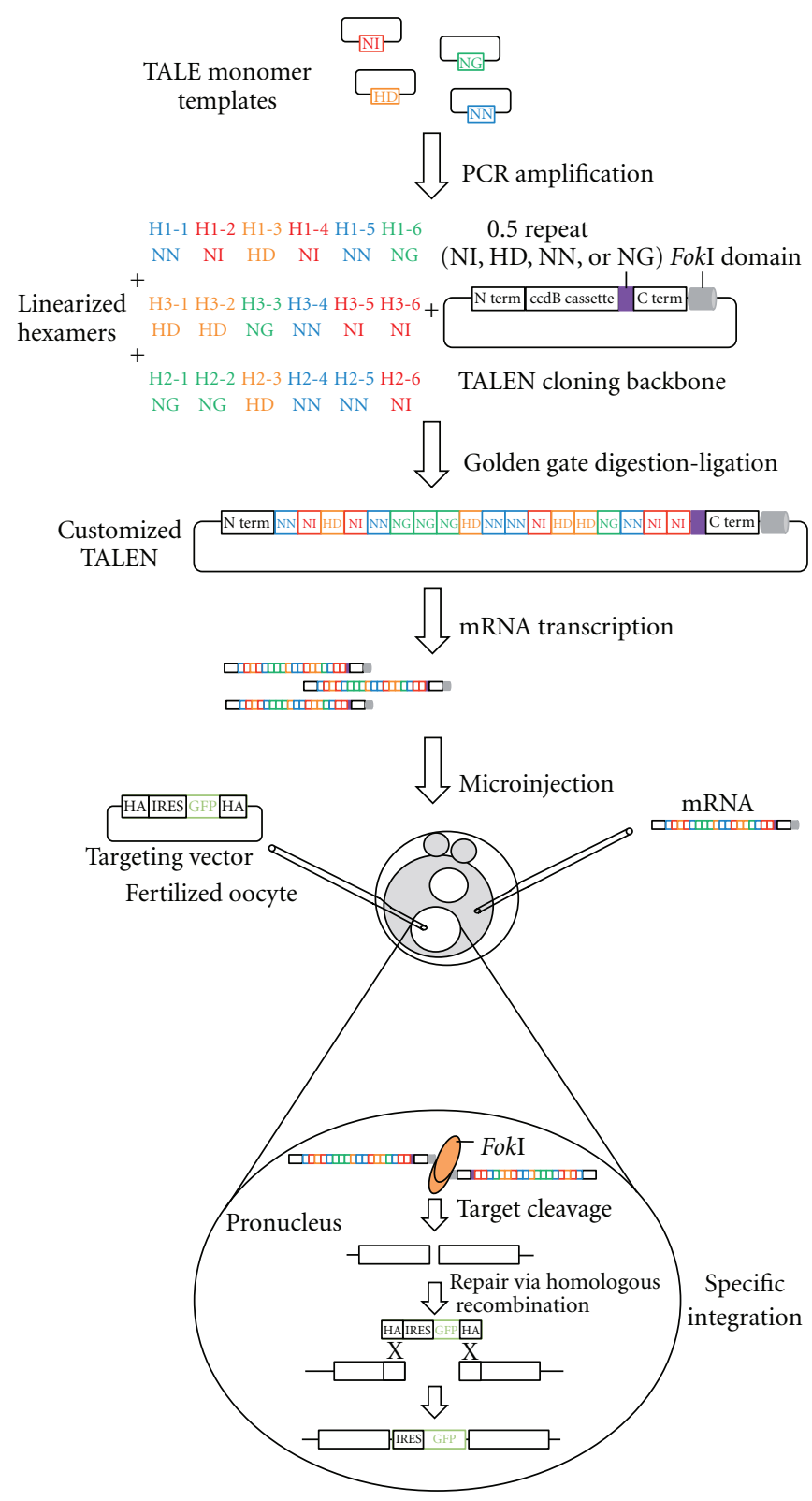

(b)

FIGURE 1: Genomic modifications through BACs and TALEN. (a) The recombination of the BAC and a transgene (GFP and the antibiotic resistance gene kanamycin) leads to a modified BAC which is then randomly integrated into the DNA after microinjection into the male pronucleus. (b) The customized TALEN, specific to the sequence of interest, is constructed by PCR and digestion-ligation of hexamers that are cloned into the TALEN backbone. Subsequently, the TALEN is transcripted into RNA followed by the microinjection of the RNA into the cytoplasm of the zygote simultaneous to the injection of the GFP containing targeting vector into the pronucleus. This results in specific integration of the GFP via homologous recombination generating a knock-in locus.

forward approach ensured that BAC transgenesis remained a viable alternative to the more complex and time-consuming targeted transgene insertion via embryonic stem cell technology [2].

Despite its increasing popularity the use of BAC transgenesis for the generation of transgenic animals is still accompanied by several problems. BAC transgenes are generated by nonspecific integration into the target genome; therefore a variable number of copies can be inserted into an unknown locus in the genome of the target organism. Moreover, because the generation of BAC transgenic founders is less efficient than conventional transgenes, it usually results in less founder lines being generated in the course of a similar microinjection effort. Furthermore, the construction of a BAC transgene can be extremely time consuming; while a BAC transgene construct may be assembled within 2 weeks, in some instances it takes several months. The construction of the BAC is then followed, depending on the facility, by 


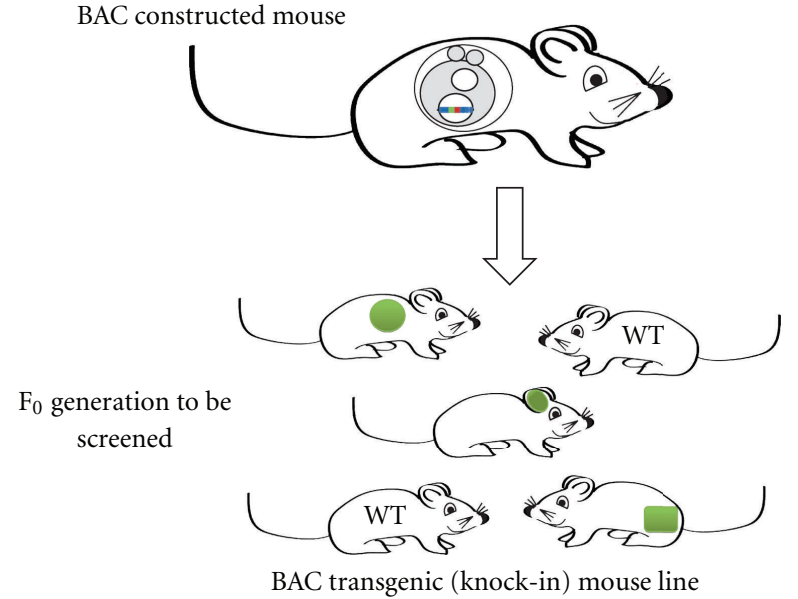

(a)

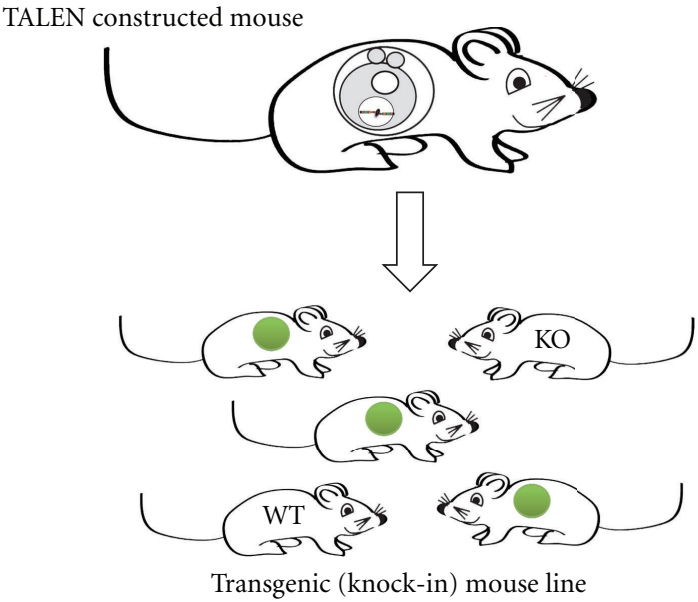

(b)

FIGURE 2: Generation of new mouse lines through BAC transgenesis or TALEN-mediated transgene insertion. (a) In consequence of the random integration of the vector during the construction of BAC transgenic mice the founders need to be screened for the appropriate expression pattern. (b) In contrast, TALEN-constructed transgenic mice only need to be checked by molecular biology methods for presence of the appropriate knock-in event. As a side product, knock-out alleles are usually generated as well.

several months of oocyte injection and screening of the founder lines (Figure 2(b)). New promising technologies have been developed which may overcome the problems associated with BAC transgenesis while retaining or even improving on its advantages over conventional transgenesis. These new techniques are based on designer nucleases and their ability to generate double strand breaks (DSBs) at a predetermined sequence in the target genome, even in the oocyte. Such DSBs can be repaired by nonhomologous endjoining (NHEJ) or homologous recombination (HR). While NHEJ results in deletions or insertions that can lead to gene deficiencies, HR can lead to the faithful integration of a coinjected targeting vector. At the time of writing, two classes of nucleases, ZFNs [3-5] and TALENs [6, 7], show the most promise of making an impact on transgenic technology in the mouse and beyond. Both assemble on the target DNA as heterodimers and each feature a DNA-binding domain designed for a specific sequence and a FokI domain containing the endonuclease activity.

The DNA-binding domains of ZFNs are assembled through multimerization of $\mathrm{Cys}_{2} \mathrm{His}_{2}$ motifs, each motif recognizing three nucleotides of the DNA. ZFNs are commonly designed with 3- to 4-zincfinger domains [8], sometimes even up to 6 fingers [9], which are then fused to the FokI domain. The ZFNs dimerize at the target sequence and cleave the double-stranded DNA thus forming DSBs. It is thought that increasing the number of ZF domains leads to an increase in the specificity and decreases the toxicity of the ZFNs. Constructs containing ZFNs encoding for a specific target can either be obtained from a commercial source such as Sigma-Aldrich or constructed in-house. The most common methods of in house construction are through open-source protocols such as Oligomerized Pool Engineering (OPEN) and Context-Dependent Assembly (CoDA) arrays. OPEN utilizes an archive of zinc finger pools specific to the sequence of interest. Multifinger arrays are then generated from these pools and arrays which have high affinity and specificity are identified via a bacterial two-hybrid system [10]. As with OPEN, CoDA is based on a library and involves cloning the 3 fingers of the ZFNs out of a large archive of $319 \mathrm{~N}$ terminal (F1) and $344 \mathrm{C}$ terminal (F3) fingers designed to work in combination with 18 fixed second fingers (F2) [11]. Compared to OPEN, this method is easier and cheaper for laboratories to adopt as no special techniques or proprietary reagents are required. CoDA does however have some drawbacks; the identity of the second finger is limited to the 18 fixed F2 units and the combined affinity or specificity of the 3 fingers in combination is not taken into account. Therefore despite the increased technical expertise required, at present OPEN may still be the most reliable choice to achieve the high specificity needed for certain therapeutic applications. Unfortunately at present both OPEN and CoDA are restricted to 3 fingers per heterodimer, thus limiting the possibility for increased specificity through inclusion of additional fingers.

TALEN nucleases are similar to ZFNs as they rely on the FokI domain for their nuclease activity. However, the DNAbinding domain is based on the TAL-like effectors originally derived from Xanthomonas plant pathogens [12]. The TAL DNA-binding domain consists of a number of repeats where one repeat recognizes one base pair of the target DNA. Each individual repeat is 33-35-amino-acids long and all repeats are essentially identical with the exception of two key amino acid in positions 12 and 13. It is these "repeat variable diresidues" (RVDs) which determine the repeat's sequence specificity. In short, the RVDs NI, HD, NN, and NG recognize A, C, G or A, and T, respectively. Since the individual repeats appear to be modular and function in a context-independent manner, it is possible to design a TALEN protein that will bind and cleave virtually any target 
sequence. As with the ZFNs two TALEN monomers are necessary to dimerize a FokI domain at the target sequence for generating a DSB $[7,13]$. Online tools are available to custom-design TALEN targeted to a specific sequence. These allow the rapid identification of a suitable target sequence and its respective TALEN pair. Several strategies for efficient generation of TALEN pairs have been published including strategies involving "Golden Gate cloning" that include the assembling of repeat modules to an intermediary array with up to 10 repeats followed by the ligation of intermediary arrays into a backbone [7] or a PCR-based protocol where the monomers of a plasmid library are amplified to first form 3 hexamers which are then ligated to one 18 mer. This protocol enables the construction of customised TALEN within few days [14] (Figure 1(b)). Several other TALEN construction systems have been published [15-17] and TALENs can also be obtained commercially.

The method of construction of TALENs and ZFNs may be different but the functional outcome of both methodologies is the same: they are efficient and reliable methods for the in vitro and in vivo production of gene deficiencies, gain of function transgenics and perhaps most promisingly new therapeutic applications. But how does this relate to BAC transgenesis? In effect, DN technology allows the insertion of reporter genes such as GFP or Cre into the gene of interest by mouse zygote injection. The ZFNs and TALENs determine the exact position for integration of the transgene via homologous recombination. To achieve this, transgene vectors containing homologous sequences, flanking the DSB as well as the transgene, are constructed and coinjected with the DN expression vectors (or their mRNA) into the zygotes. This results in integration similar to that achieved by time-consuming embryonic stem (ES) cell culture, yet obtained with the speed of conventional transgenesis and without using selection genes such as neomycin resistance. Moreover, the founder lines of transgenic animals generated through BAC technology have to be functionally screened thoroughly due to the expression variegation as result of the randomness of the integration and an appropriate line has to be identified. In the case of DN-assisted HR all founder lines are carrying identical gene modifications and they can all be used for functional confirmation and analysis (Figure 2). While the efficiency of TALENs in mouse zygotes still needs to be evaluated, ZFNs have already been shown to allow efficient integration of DNA into a target site such as the frequently used gt(ROSA)26Sor locus $[5,18]$. Thus, it may be envisioned that in the future, BAC transgenes will not be used to drive transgenesis but instead functional transgenes will be directly integrated into endogenous loci. As with insertion transgenes involving ES cell technology, this may be achieved by either integration (or mutation) of the start ATG, leading in most cases to a functional knockout allele, or by placing the transgene in the $5^{\prime}$ untranslated region and using an internal ribosomal entry site (IRES) or 2A element thus maintaining the open reading frame of the "hijacked" gene. Moreover placement of open reading frames under the control of loxP-flanked STOP cassettes or even exogenous promoters through placement of the respective constructs in the target locations may also be envisaged. As a side product these approaches would in many cases also yield deficiency alleles after faulty NHEJ repair.

Though it may appear that the advent of DNs will make BAC transgenesis all but obsolete, applications for BAC transgenes remain. Detailed functional analysis of human genetic elements in model organisms such as the mouse is still likely to be carried out using BAC transgenesis. Also, BACs may still serve as donors for homology regions. Additionally, although this paper highlights some of the future possibilities of genomic manipulation through DNs, many obstacles both small and large still remain. These include the determination of offsite effects (mutations) and what outcomes they may have. Furthermore a gold standard protocol for identification of target sequences and DNs must still be validated in vivo and other considerations such as the optimum length of homology regions for integration at DSBs in oocytes, and whether the targeting vector should be supplied in linear or circular form must still be decided. Only then a full financial and scientific comparison of BAC technology versus insertion through use of DNs will be possible.

Taken together, this paper has discussed some of the advantages and disadvantages of BAC technology in the context of the newly evolving DN technology which allows targeted insertions in the oocyte. We believe this is a revolutionary time in transgenic technologies and that the use of DN technology could make not only ES cell culture techniques but also conventional transgenesis, including BAC transgenesis all but obsolete.

\section{References}

[1] H. Shizuya, B. Birren, U. J. Kim et al., "Cloning and stable maintenance of 300-kilobase-pair fragments of human DNA in Escherichia coli using an F-factor-based vector," Proceedings of the National Academy of Sciences of the United States of America, vol. 89, no. 18, pp. 8794-8797, 1992.

[2] T. Johansson, I. Broll, T. Frenz et al., "Building a zoo of mice for genetic analyses: a comprehensive protocol for the rapid generation of BAC transgenic mice," Genesis, vol. 48, no. 4, pp. 264-280, 2010.

[3] H. J. Lee, E. Kim, and J. S. Kim, "Targeted chromosomal deletions in human cells using zinc finger nucleases," Genome Research, vol. 20, no. 1, pp. 81-89, 2010.

[4] C. Öllü, K. Pars, T. I. Cornu et al., "Autonomous zinc-finger nuclease pairs for targeted chromosomal deletion," Nucleic Acids Research, vol. 38, no. 22, pp. 8269-8276, 2010.

[5] M. Meyer, M. H. De Angelis, W. Wurst, and R. Kühn, "Gene targeting by homologous recombination in mouse zygotes mediated by zinc-finger nucleases," Proceedings of the National Academy of Sciences of the United States of America, vol. 107, no. 34, pp. 15022-15026, 2010.

[6] F. Zhang, L. Cong, S. Lodato, S. Kosuri, G. M. Church, and P. Arlotta, "Efficient construction of sequence-specific TAL effectors for modulating mammalian transcription," Nature Biotechnology, vol. 29, no. 2, pp. 149-154, 2011.

[7] T. Cermak, E. L. Doyle, M. Christian et al., "Efficient design and assembly of custom TALEN and other TAL effector-based constructs for DNA targeting," Nucleic Acids Research, vol. 39, no. 12, article e82, 2011. 
[8] J. Wu, K. Kandavelou, and S. Chandrasegaran, "Customdesigned zinc finger nucleases: what is next?" Cellular and Molecular Life Sciences, vol. 64, no. 22, pp. 2933-2944, 2007.

[9] D. J. Segal, J. W. Crotty, M. S. Bhakta, C. F. Barbas 3rd, and N. C. Horton, "Structure of Aart, a designed six-finger zinc finger peptide, bound to DNA," Journal of Molecular Biology, vol. 363, no. 2, pp. 405-421, 2006.

[10] M. L. Maeder, S. Thibodeau-Beganny, J. D. Sander, D. F. Voytas, and J. K. Joung, "Oligomerized pool engineering (OPEN): an "open-source" protocol for making customized zinc-finger arrays," Nature Protocols, vol. 4, no. 10, pp. 1471-1501, 2009.

[11] J. D. Sander, E. J. Dahlborg, M. J. Goodwin et al., "Selectionfree zinc-finger-nuclease engineering by context-dependent assembly (CoDA)," Nature Methods, vol. 8, no. 1, pp. 67-69, 2011.

[12] J. Boch, H. Scholze, S. Schornack et al., "Breaking the code of DNA binding specificity of TAL-type III effectors," Science, vol. 326, no. 5959, pp. 1509-1512, 2009.

[13] J. C. Miller, S. Tan, G. Qiao et al., "A TALE nuclease architecture for efficient genome editing," Nature Biotechnology, vol. 29, no. 2, pp. 143-148, 2011.

[14] N. E. Sanjana, L. Cong, Y. Zhou, M. M. Cunniff, G. Feng, and F. Zhang, "A transcription activator-like effector toolbox for genome engineering," Nature Protocols, vol. 7, no. 1, pp. 171192, 2012.

[15] J. D. Sander, L. Cade, C. Khayter et al., "Targeted gene disruption in somatic zebrafish cells using engineered TALENs," Nature Biotechnology, vol. 29, no. 8, pp. 697-698, 2011.

[16] T. Li, S. Huang, X. Zhao et al., "Modularly assembled designer TAL effector nucleases for targeted gene knockout and gene replacement in eukaryotes," Nucleic Acids Research, vol. 39, no. 14, pp. 6315-6325, 2011.

[17] D. Reyon, S. Q. Tsai, C. Khgayter, J. A. Foden, J. D. Sander, and J. K. Joung, "FLASH assembly of TALENs for high-throughput genome editing," Nature Biotechnology, vol. 30, no. 5, pp. 460465, 2012.

[18] M. Hermann, M. L. Maeder, K. Rector et al., "Evaluation of OPEN zinc finger nucleases for direct gene targeting of the ROSA26 locus in mouse embryos," PLoS ONE. In press. 

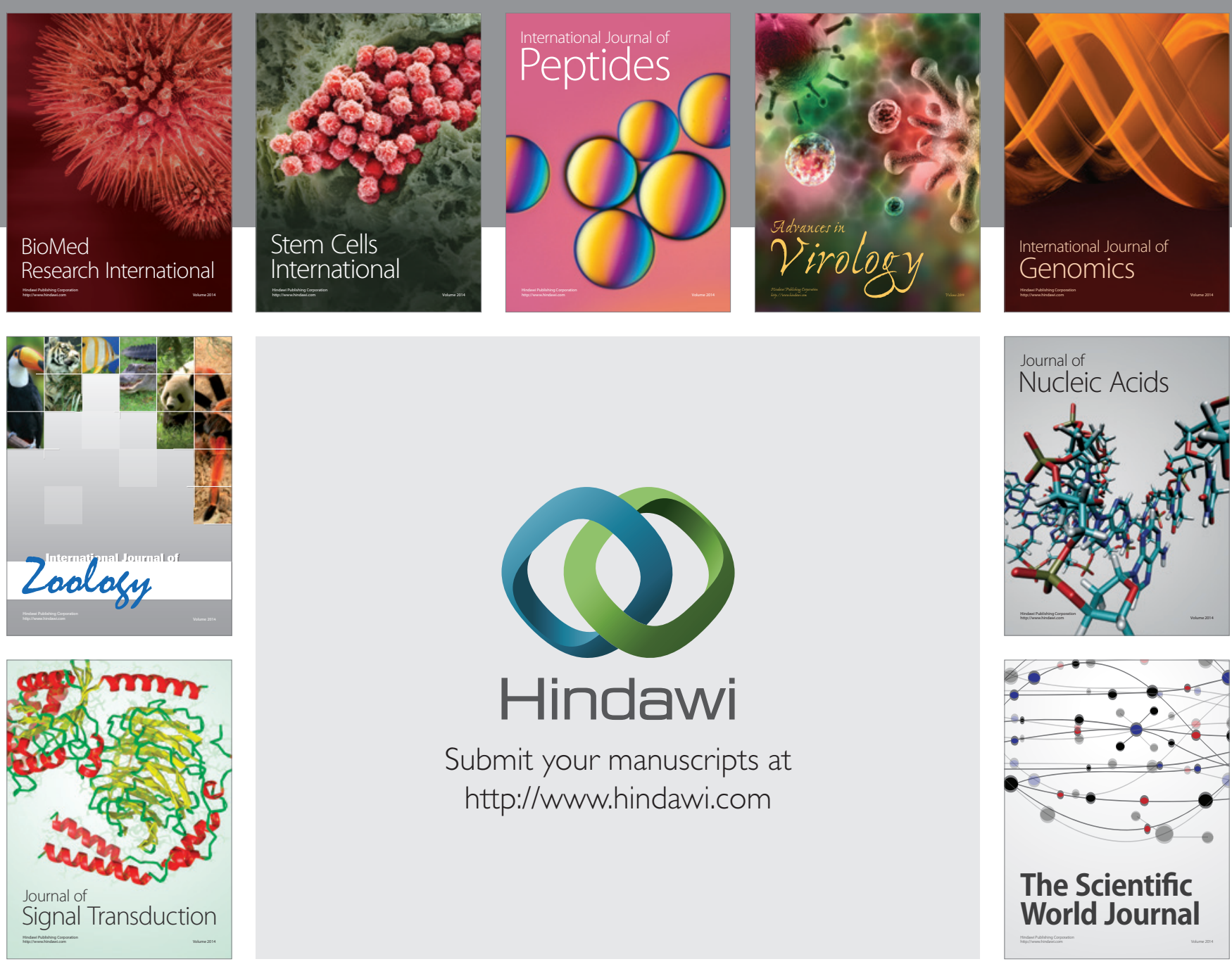

Submit your manuscripts at

http://www.hindawi.com
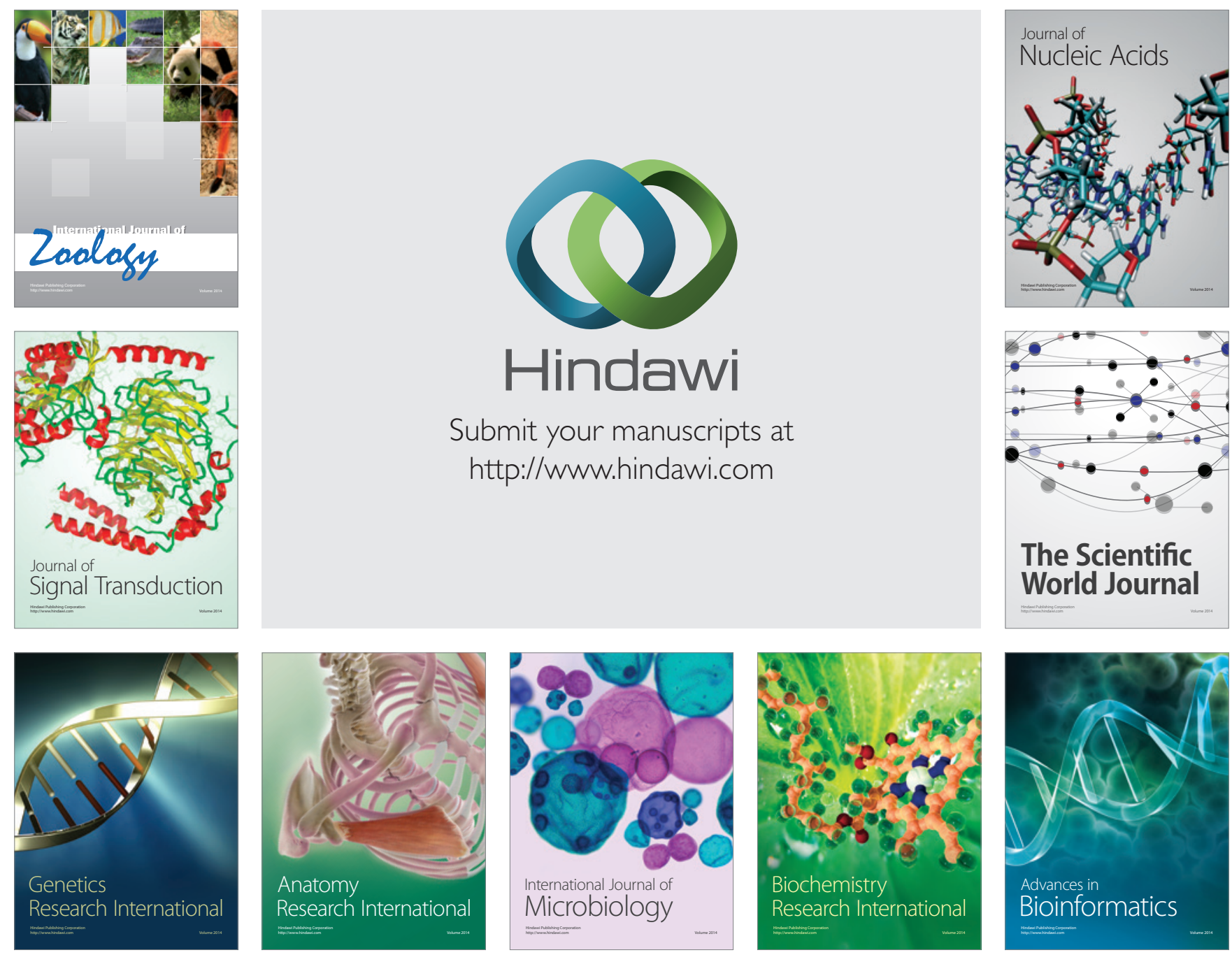

The Scientific World Journal
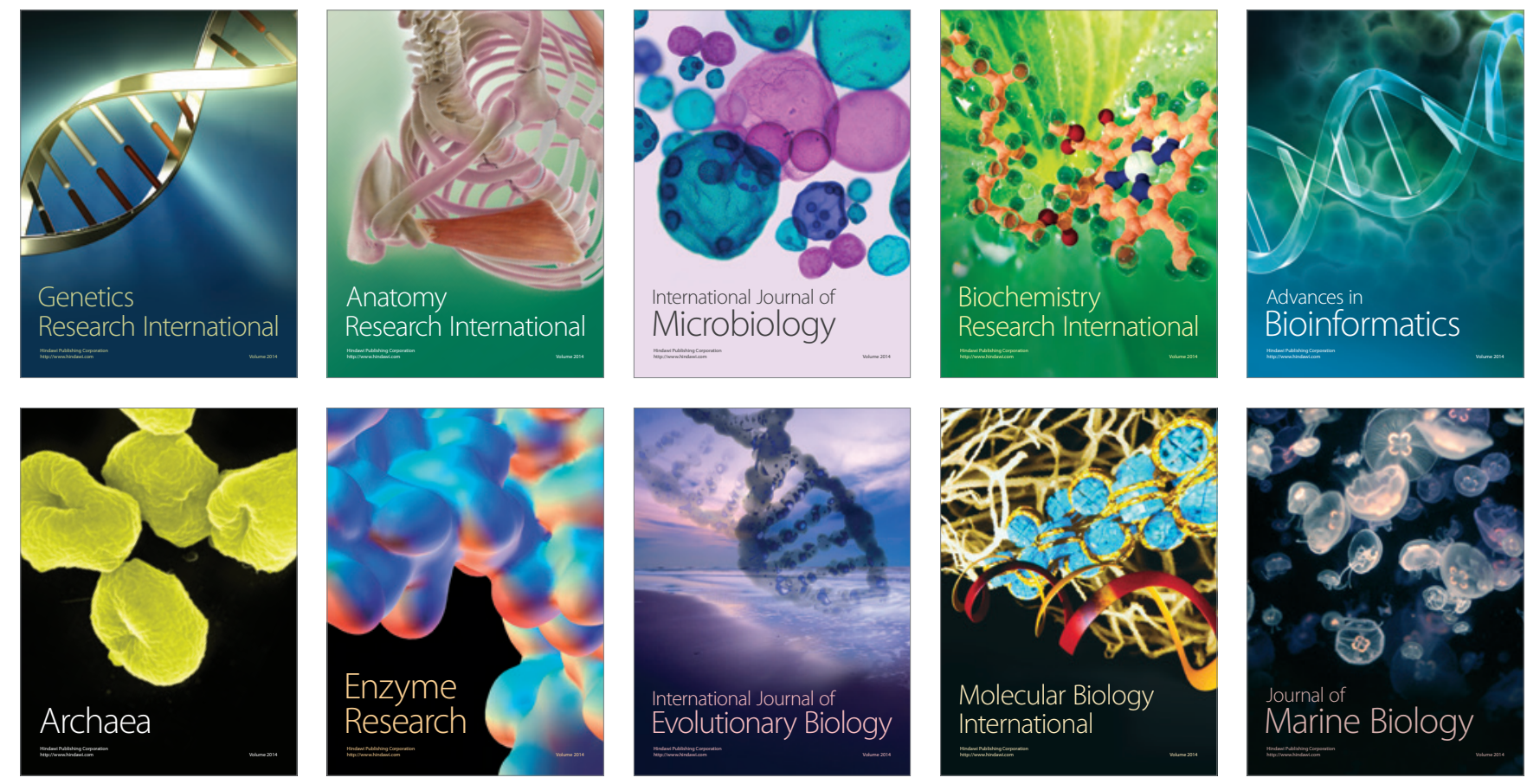